\title{
La narrativa como herramienta didáctica y de comunicación para la enseńanza de la historia social contemporánea y reciente
}

\section{Narrative as a didactic and communication tool for the teaching of the contemporary and recent social history}

Renée Isabel Mengo

Doctora en Comunicación Social (Universidad de La Laguna, Tenerife, España). Licenciada y profesora en Historia, (Universidad Católica de Córdoba). Docente adjunta en la Cátedra de Historia Social Contemporánea de la ECI-UNC. Docente adjunta por concurso en la Facultad Regional Córdoba de la Universidad Tecnológica Nacional.

Contacto: rimm952@gmail.com

Pablo Rubén Tenaglia

Becario Interno de Finalización de Doctorado (CONICET).

Doctorando en Estudios Sociales de América Latina, línea Análisis Interdisciplinario en Historia y Política

Contemporánea (CEA-UNC) Licenciado en Comunicación Social. (ECI-UNC).

Docente asistente en la Cátedra de Historia Social Contemporánea y asistente por concurso en el Taller de Práctica

Docente III de la Licenciatura y del Profesorado Universitario en Comunicación Social. (ECI-UNC).

Contacto: pablotenaglia2001@yahoo.com.ar

Artículo de investigación

Fecha de recepción: marzo 15 de 2015 • Fecha de aprobación: abril 6 de 2015

\section{RESUMEN}

Este artículo tiene como objeto relatar aspectos desarrollados en el curso de extensión "Documentación narrativa de experiencias pedagógicas significativas", dictado en el año 2014 en la Escuela de Ciencias de la Información de la Universidad Nacional de Córdoba, Argentina, y describir la nueva edición del curso en el año 2015, que abordará específicamente las líneas temáticas de las narrativas en la enseñanza de la historia social contemporánea y reciente, y las narrativas de experiencias en el uso de las nuevas tecnologías, contribuyendo así a la democratización de la construcción del conocimiento mediante el empleo de recursos didácticos y de la comunicación. 
Palabras clave: narrativas, historia contemporánea, herramientas, comunicación, enseñanza.

\begin{abstract}
This article aims to describe aspects developed in the course of Extension: Significant Educational Experiences of Narrative Documentation, dictated in 2014 at the School of Information Sciences at the National University of Cordoba-Argentina, as well as to describe the new edition of the course in 2015, which will address specifically the thematic lines of narrative in the teaching of contemporary and recent social history, and the narratives of experiences in the use of new technologies, contributing this way to the democratization of knowledge construction by the use of teaching resources and communication.
\end{abstract}

Keywords: Narratives, contemporary history, tools, communication, teaching.

\title{
INTRODUCCIÓN
}

En la sociedad del conocimiento, las nuevas tecnologías de la información y la comunicación (TIC) transversalizan el desarrollo de todas las actividades. La escuela como institución básica y prioritaria de la sociedad, y la universidad como ámbito insoslayable de formación y capacitación profesional no escapan a este dinámico y acelerado cambio que comenzó en las últimas décadas del siglo XX y tiende a profundizarse en el siglo XXI. En este nuevo contexto los estudiantes, docentes y directivos llevan a cabo sus prácticas, se planifica, se enseña y se evalúa... Pero, ¿por qué no narrar esas prácticas?, ¿̨por qué no socializar nuestras experiencias?, ¿̨por qué no hacernos un tiempo dentro de la vorágine del día a día en la tarea docente, para dar la posibilidad de que nuestra tarea se conozca y circule más allá de nuestra escuela...?

Con el presente artículo se busca relatar y fundamentar la experiencia llevada a cabo en el año 2014 en la Escuela de Ciencias de la Información de la Universidad Nacional de Córdoba en donde, a partir de un curso de extensión titulado "Documentación narrativa de experiencias pedagógicas significativas", se materializó la propuesta en la publicación de un libro, en el que con trece relatos de diez docentes y dos directivos 
(uno de nivel secundario y otro de un Instituto Superior de Formación Docente), y con base en la participación estudiantil se propició la narración, documentación y sistematización de experiencias pedagógicas significativas, entendiendo estas como herramientas didácticas válidas y de comunicación para la enseñanza, ya que son antecedentes de buenas prácticas y actos de gestión dados en diferentes contextos educativos reales, todos ellos tendientes a profesionalizar la tarea docente y directiva, en pos de una mejor escuela o universidad a partir de la circulación de esta información empírica, utilizando los diversos medios que las nuevas tecnologías posibilitan.

Es de destacar también que la publicación fue fruto del trabajo colaborativo y esfuerzo en equipo, realizado por los cursantes del citado taller de formación docente, los cuales provenían en su mayoría de la rama de la comunicación (tanto Licenciatura como Profesorado) y otros, de diferentes ámbitos, pero todos con el denominador común de ser estudiantes, docentes o directivos. Se aclara que el curso de extensión se basó en un proyecto de capacitación aprobado por la Escuela de Ciencias de la Información con resolución 481/2013 y por la Red de Formación Docente Continua de la Subsecretaría de Estado de Promoción de Igualdad y Calidad Educativa de la Provincia de Córdoba con Resolución de aceptación 101/2014 y de aprobación $496 / 2014$.

El proceso de publicación de las narrativas fue posible gracias a un articulado y responsable trabajo colaborativo de docentes y estudiantes donde los mismos fueron los protagonistas de las correcciones, sugerencias y observaciones de todos los relatos, esto a través del uso de las nuevas tecnologías de la información y la comunicación, utilizando redes sociales como Facebook, correo electrónico y la aplicación WhatsApp en teléfonos móviles.

El uso de las TIC favoreció la dinámica y metodología del proceso de evaluación de escritos propiciando así un aprendizaje colaborativo que fue surcado por espacios de crítica y debate entre los cursantes.

Con base en la fructífera experiencia citada y llevada a cabo en el año 2014, se proyecta para el presente ańo ampliar la propuesta, profundizando la temática con la edición de dos libros en los que se trabajará nuevamente con las narrativas como herramientas 
didácticas de comunicación para la enseñanza en dos líneas: una, narrativas pedagógicas sobre el uso de las TIC y la otra, sobre narrativas pedagógicas en la enseńanza de la Historia social contemporánea. Estas dos líneas surgieron de la motivación y sugerencia de los cursantes en el año 2014, quienes demandaron profundizar y actualizar el contenido del curso en las dos grandes temáticas de su interés.

Para la nueva propuesta, titulada: "Documentación narrativa y registros pedagógicos sobre el uso de las TIC en la gestión directiva y práctica docente" se sumarán como profesores invitados, Supervisores de Nivel Secundario tanto de Córdoba capital como del interior y docentes de Historia contemporánea que forman parte de la propuesta de postgrado de esta unidad académica (Diplomatura y Doctorado).

\section{¿POR QUÉ NARRAR EXPERIENCIAS DE PRÁCTICA DOCENTE Y DE GESTIÓN DIRECTIVA?}

La importancia que tiene la documentación narrativa de nuestras prácticas y actos de gestión como también de la participación estudiantil en actividades formativas, hace que a partir de su elaboración se propicie la realización de una trama de fortalecimiento, sistematización y recuperación de las propias experiencias de los docentes, estudiantes y directivos que cuentan historias de un contexto educativo concreto, elaborando relatos de un enorme potencial pedagógico que sirve como insumo a sus propios pares para interpretar el mundo escolar desde el punto de vista de sus protagonistas, haciendo posible así la elaboración de estrategias de intervención y de investigación pedagógica sostenidas y pertinentes. Se entiende así, que estas narraciones son útiles herramientas didácticas y de comunicación para fortalecer procesos pedagógicos a futuro.

Estas narraciones son más interesantes aun, cuando los propios estudiantes y docentes, a partir de un trabajo colaborativo y utilizando las nuevas tecnologías de la información y la comunicación (TIC) hacen su propia edición y sugerencias de corrección a sus compañeros. Este proceso en donde se leen experiencias de terceros, se redacta la propia, se observan y corrigen las del grupo, se edita el material y se diseña la publicación, es un excelente proceso de capacitación entre pares que se 
debería permitir en cualquier universidad, instituto de formación docente o escuela en diferentes jornadas institucionales de reflexión. Con ello es posible relatar situaciones concretas que escapan a lo dicho, a lo oficial, a lo prescripto...

“(...)Es así como, el registro, la sistematización escrita, el acopio y la difusión pública de experiencias, prácticas y saberes escolares contadas a través de la voz y la palabra de los docentes constituyen, al mismo tiempo, una propuesta político pedagógica para la escuela y un programa para el desarrollo profesional docente entre docentes. En efecto, al estimular y garantizar procesos de escritura, lectura, reflexión e interpretación pedagógica de las propias experiencias escolares de los docentes, se espera no sólo resguardar y divulgar un corpus de documentos y conocimientos distintos a los habituales, sino también hacer posibles experiencias de formación horizontal que signifiquen el desarrollo profesional de los involucrados y una contribución para la mejora y transformación de las prácticas de la escuela. En este caso, la innovación pedagógica se inclina por potenciar la productividad cultural e imaginación didáctica de los docentes y, en ese mismo movimiento, se proclama política y los afirma como protagonistas de la vida y la transformación escolar. Al mismo tiempo que promueve el cambio, se reconoce en la tradición pedagógica y la cultura escolar, conversa con sus palabras y tensiona sus saberes. Por eso, una vez planteada la necesidad de mirar y reconocer a las instituciones educativas y a las escuelas desde otro enfoque, lo que sigue pretende acompañar y profundizar esa perspectiva (...)" (Suárez, 2005, p. 22).

\section{LAS NARRATIVAS COMO HERRAMIENTAS DIDÁCTICAS Y DE COMUNICACIÓN PARA LA ENSEÑANZA}

Según el uso del término coloquial, una herramienta es un objeto elaborado a fin de facilitar la realización de una actividad cualquiera sea esta. Cuando hablamos de herramientas didácticas y de comunicación, indefectiblemente estos instrumentos son elaborados para facilitar la interacción humana en el plano pedagógico y educativo. 
La narrativa de experiencias pedagógicas funciona como una herramienta válida de comunicación para la enseńanza, ya que su esencia horizontal ligada a que esos relatos, provienen de la misma práctica docente, sin haber mediado la intervención estatal a través de prescripciones de diseños curriculares sirve como insumo de valor para los propios pares que al leer esas situaciones reales, dadas en contextos educativos determinados, pueden aplicarlas en el desarrollo de sus propias prácticas o actos de gestión.

Un buen uso didáctico de diferentes recursos áulicos o herramientas de enseñanza (en este caso las narrativas) enriquece los procesos de enseñanza aprendizaje, ya que se pueden aplicar en situaciones determinadas como las que se exigen en una sociedad de información y conocimiento como en la que vivimos.

Gustavo Bombini, profesor de la cátedra de Didáctica de la Lengua y la Literatura y Prácticas de la Enseñanza de la carrera de Letras en la Universidad Nacional de La Plata, hace una descripción interesante de estas narraciones como herramientas didácticas y de comunicación aplicadas a la enseñanza. En el tercer capítulo, Narrar las prácticas de su libro "Reinventar la enseñanza de la lengua y la literatura", comienza con varios relatos donde distintos docentes dan cuenta de situaciones por demás interesantes con sus estudiantes en el desarrollo de sus clases.

Sucede que estos relatos producto de intercambios más o menos informales entre colegas, están mostrando la potencia y la diversidad de unos modos de hacer que están transcurriendo y que van tramando un campo difuso pero rico, opulento, en prácticas posibles, atravesado por reflexiones de esas que se hacen ahí, al borde de la experiencia de enseñar (...)

Repensar los problemas de la enseñanza, construir una didáctica, elaborar un currículo de una disciplina específica, exige la consideración de estas historias, el reconocimiento en estas narraciones "de un saber acumulado que tiene que ver con los modos de resolver los desafíos de la enseñanza (Bombini, 2006, p. 85). 
De este modo la narración de experiencias pedagógicas significativas y la escritura se transforma en un modo de reflexionar y de producir conocimiento acerca de la práctica por el mismo docente o estudiante que vivenció esa experiencia.

La narración de experiencias pedagógicas significativas como herramientas de comunicación aplicadas a la enseńanza, también propicia una democratización de esa construcción de conocimiento tradicional y solo permitida a saber para los teóricos o técnicos que muchas veces están lejos de un aula y de todas las situaciones que en ella suceden.

\section{LAS NARRATIVAS EN LA ENSEÑANZA DE LA HISTORIA SOCIAL CONTEMPORÁNEA Y RECIENTE}

Con base en la experiencia llevada a cabo en el año 2014, donde se trabajaron las narrativas como generalidad en el empleo de estas como herramientas didácticas y de comunicación aplicadas a la enseñanza, y a partir de la sugerencia de los cursantes, en el taller que se proyecta para este ańo se profundizará la temática en dos líneas, las narrativas de experiencias pedagógicas en el uso de las nuevas tecnologías de la información y la comunicación (TIC) y las narrativas como herramientas didácticas y de comunicación para la enseñanza de la historia contemporánea y reciente.

Para abordar la disciplina de la historia, a partir de consultas a especialistas en este campo y conjuntamente a las demandas de los estudiantes, se optó por trabajar con la historia más cercana a nuestros días, conocida como historia contemporánea y reciente, la que se describe a continuación.

Se debe tener presente que en los últimos años estamos asistiendo al nacimiento de un nuevo campo de estudios: la historia reciente. Aunque siempre hubo historiadores que se ocuparon de describir y analizar procesos históricos de su tiempo, lo novedoso está en que solo en los últimos años "se constituye un campo de estudios con problemáticas propias” (Franco y Levin, 2007, p. 16). 
Por ello, cobra importancia como alternativa y desafío de cambio didáctico y de comunicación, la enseñanza de la historia reciente, la que interfiere con el deber de la memoria que se le ha puesto a la escuela y a la universidad. "En la clase de historia no se recuerda, se reconstruye", sostiene la especialista Kriger (2011). El docente desde su permanente actualización, debe reconocer los modos en que las narrativas escolares de la historia reciente han variado en las últimas tres décadas, y por lo tanto, su relación con la construcción de memorias sociales.

Enseñar la historia reciente a partir de las narrativas conduce a plantear y resolver problemas y cuestiones pertinentes para su abordaje, como interrogantes y conflictos, lo sostiene Carnovale (2007) ya sea en aportes para pensar en su enseńanza, tanto como la articulación entre testimonios, contextos y conceptos. La explicación histórica y su complejidad, es la gran contribución que con profesionalismo y compromiso puede aportar la historia contemporánea y reciente.

La misma, considerada como "un campo en construcción" (Franco y Levin, 2007, p. 55) y "epistemológicamente inestable", toma un lugar central en su delimitación por la cercanía temporal. La cercanía misma, establecida por el uso de criterios simplemente cronológicos o, más complejamente, la cercanía que se expresa en la coetaneidad entre pasado y presente, por la supervivencia de quienes protagonizaron esa historia, la existencia de una memoria social viva sobre ese pasado o la contemporaneidad entre el historiador y su objeto de investigación, la hacen un rico campo a investigar en la era digital en la que las nuevas tecnologías de la información y comunicación transverzalizan cualquier disciplina.

Cabe aquí plantear si la historia social contemporánea y reciente analiza procesos históricos inacabados, en desarrollo, cómo algunos de esos procesos pueden extenderse por décadas (y aun siglos), la cotidianeidad entre el pasado y presente no se limita a los ańos recientes. Por tanto, el problema no se presenta solo a quienes investigan el hoy. El temor al sesgo de la subjetividad y la ideología que se presentan a la historia reciente como campo de investigación, también aparece con relación a las fuentes de donde se recoge la información. Esta historia como toda investigación histórica, se sustenta en documentos, fuentes hemerográficas, pero también en el testimonio. 
Es ahí donde las narrativas toman sentido, tanto desde las que pueden elaborar los estudiantes como las que son redactadas por los propios docentes y aun directivos.

\section{LAS TIC Y LA HISTORIA SOCIAL CONTEMPORÁNEA Y RECIENTE}

En este contexto, las TIC pueden tener un papel protagónico en el aporte de las prácticas; a través de las nuevas tecnologías es posible acercar documentos reales al trabajo diario del aula, trabajar con audiovisuales que recreen procesos históricos, hacer reconstrucciones virtuales de restos arqueológicos o de situaciones históricas, entre otros. A su vez, las TIC e Internet nos permiten trabajar colaborativamente con otros centros (escuelas, liceos, universidades, archivos históricos, etc.), obtener imágenes, materiales, reconstrucciones, documentos, archivos, etc. Se cuenta, además, con la posibilidad del trabajo colaborativo entre alumnos de la misma escuela, universidad y de otros centros educativos. Los nuevos entornos de campus virtual permiten nuevos estilos de trabajo, en el aula, y sobre todo, fuera de ella.

La introducción de nuevas tecnologías ha facilitado el ejercicio de la enseñanza y ha abierto un amplio espectro de técnicas pedagógicas y fuentes de información. El problema ya no es la escasez de medios sino el exceso de conocimientos plasmados en el mundo virtual, lo que debe conducir a la selección de contenidos especializados y variados para las clases. Ante este hecho, los alumnos deben desarrollar un espíritu crítico que los lleve a juzgar las fuentes pertinentes y a reflexionar sobre su lugar en el mundo, sobre la sociedad actual y sobre cómo debe proyectarse esta hacia el futuro.

El punto neurálgico de este proceso reside en escoger las TIC adecuadas para cada trayecto pedagógico. Si partimos de la premisa de que cada área del conocimiento es diferente, ¿cuáles son las TIC que se necesitan para la clase de historia social contemporánea y reciente? La práctica ha hecho muy conocidas a los moodle, los blogs, YouTube y hasta Google Earth, que son herramientas que se utilizan con frecuencia en el proceso de aprendizaje.

En síntesis, la introducción de las TIC en la enseñanza de la historia contemporánea y reciente debe conducir hacia un aprovechamiento a fondo de los recursos que ofrece 
la red y a una innovación en la presentación de los resultados del proceso formativo. La historia, mediante las nuevas tecnologías, debe descender del pedestal de la academia y hacerse más comprensible para la sociedad, su verdadera protagonista; es aquí donde las narrativas aportan testimonios significativos en el uso de las nuevas tecnologías como en la enseñanza de la historia contemporánea y reciente.

La pedagogía requiere innovación, análisis crítico, reflexión, apertura a nuevos conocimientos y una carta de navegación que fije las reglas del juego, tan importante como la revisión y búsqueda de nuevos paradigmas. En todos los casos, la tecnología cumple un papel fundamental en la enseñanza, la cual debe ser dinámica y coherente con las transformaciones de la sociedad.

Acorde al momento cultural del presente, las TIC se han constituido como herramientas sumamente útiles para el desarrollo de la investigación y la enseñanza de las ciencias sociales, y puede decirse que la "cibercultura" ha llegado a todos sus ámbitos. Los avances en los estudios humanísticos ahora pueden ser compartidos por diferentes expertos, hecho que enriquece el análisis social y conduce a la generación de proyectos conjuntos entre centros de pensamiento de diversos países, lo que conforma una verdadera "sociedad del conocimiento".

La conexión global entre diferentes nodos de información ha permitido el acceso a fuentes imprescindibles para el estudio de los fenómenos sociales e históricos; por ejemplo, ahora es posible consultar documentación ubicada en otros territorios, gracias a la digitalización y al almacenamiento virtual de innumerables fondos de archivos y bibliotecas internacionales, otrora inalcanzables por cuestiones económicas. Por otro lado, las bases de datos bibliográficas situadas en Internet facilitan la consulta de materiales de revistas académicas y tesis universitarias.

\section{Apostemos a narrar en el CONTEXTo de las NueVas TeCNOlogías EN LA ESCUEla}

Continuando con la temática del curso dado y con lo que se proyecta trabajar en esta nueva edición, las narraciones no son solo interesantes para trabajar como 
herramientas didácticas y de comunicación en la enseñanza, también lo pueden ser para el aprendizaje, más aún en el contexto de lo que llamamos la "Escuela TIC", esta, que es transverzalizada por los medios de comunicación y todas las herramientas y recursos que de ellos devienen.

Como se dijo anteriormente estas narraciones producidas por estudiantes, docentes y directivos son insumos para la enseńanza ya que como construcción de conocimiento empírico basado en situaciones reales y caracterizadas por la horizontalidad en función de la no intervención de prescripciones curriculares, también se pueden trabajar con los alumnos desde el aula a partir del trabajo colaborativo en el marco del uso de las TIC.

La escuela TIC se enmarca en una sociedad nueva, en la que el conocimiento y la información priman. Castells (2002) describe a estas sociedades:

(...)Precisando un poco más, se trata de una sociedad en la que las condiciones de generación de conocimiento y procesamiento de información han sido sustancialmente alteradas por una revolución tecnológica centrada sobre el procesamiento de información, la generación del conocimiento y las tecnologías de la información. Esto no quiere decir que la tecnología sea lo que determine; la tecnología siempre se desarrolla en relación con contextos sociales, institucionales, económicos, culturales, etc. Pero lo distintivo de lo que está pasando en los últimos diez o quince años es realmente un paso paradigma muy parecido al que ocurrió cuando se constituyó la sociedad industrial —y no me refiero simplemente a la máquina de vapor, primero, y a la electricidad, después- Se constituye un paradigma de un nuevo tipo en el que todos los procesos de la sociedad, de la política, de la guerra, de la economía pasan a verse afectados por la capacidad de procesar y distribuir energía de forma ubicua en el conjunto de la actividad humana (...) (p. 2).

En el contexto de esta sociedad y esta nueva escuela, el trabajo con los estudiantes resulta interesante cuando se puede desarrollar de manera original y propiciando el aprendizaje colaborativo a partir del uso de las TIC. La propuesta de que elaboren 
narraciones es interesante, más cuando uno entiende qué tipo de perfil de los mismos hay en la actualidad y cuáles son sus verdaderas necesidades.

Los estudiantes de hoy -nativos digitales- según Prensky (2001) están acostumbrados a recibir información muy rápido. Les gusta realizar en paralelo múltiples tareas, prefieren ver imágenes antes que libros de texto, optan por el acceso al azar a la información -como en los hipertextos-, trabajan mejor cuando están interconectados y disfrutan de los trabajos planteados como juegos. Frente a este panorama dominado por una dinámica de continuos cambios, los docentes -inmigrantes y colonos digitales- (Pedró, 2008), deben mantenerse constantemente actualizados y poner en juego toda la creatividad, destreza y capacidad inventiva para diseñar estrategias exitosas de incorporación de TIC en las actividades del aula (Pedró, 2012).

La sociedad digital, definida por la explosión de las nuevas tecnologías de la comunicación, es, entre otras cosas, global, interactiva, caótica y difícil de controlar; los enseñantes deben asumirlo y jugar con estos caracteres a su favor, en todo caso, debe aprender a servirse de la tecnología en su beneficio, pero, sobre todo, a cambiar de mentalidad e interiorizar una nueva visión del mundo.

Tal como lo sostiene Sunkel (2008) "En cuanto a la enseñanza, el mayor desafío didáctico es la adaptación mental y el cambio generacional de los docentes en el uso de las nuevas tecnologías. Asimismo, esta adaptación debe ir acompañada de un riguroso y planificado proceso de revisión y selección de los diseños curriculares, como de la problematización de los núcleos prioritarios de aprendizaje, partiendo de la revisión permanente de los paradigmas que representan a este particular momento, en donde la flexibilidad forma parte del común denominador de las ciencias..." Qué mejor panorama que este para poder trabajar con narrativas de experiencias pedagógicas, tanto de docentes como de estudiantes.

En paralelo, para el Estado, otro desafío en la implementación de una nueva didáctica es la inversión en capacitación de esta nueva forma de enseñar y de aplicar nuevos recursos instrumentales de mayor dinamismo para generar un cambio actitudinal con respecto a la tradicional forma de dar enfoque a la enseñanza (González, Mengo, Pizarro \& Tenaglia, 2014, p. 75). 


\section{NUESTRA EXPERIENCIA DE APRENDIZAJE COLABORATIVO EN EL AÑo 2014}

Muchas veces se enseña desde la teoría, pero llevar esos aportes a la práctica cierta y concreta resulta difícil de aplicar. Diferentes situaciones, ya explicitadas anteriormente, como la vorágine en la que vivimos, acelerada por la interconexión de ámbitos y recados solicitados a través de los diferentes dispositivos de comunicación, hacen que se prioricen otras tareas, que se vean como lejanas las posibilidades de cristalizar los objetivos.

El esfuerzo conjunto y el trabajo en equipo de manera colaborativa en el curso de Documentación narrativa de experiencias pedagógicas significativas del año 2014, hicieron posible que este grupo de profesionales de la comunicación y la educación pudieran hacer realidad documentar y sistematizar experiencias.

Uno de los aspectos más fuertes de esta publicación fue el trabajo de autocorrección y evaluación de los diferentes escritos que realizaron los mismos cursantes entre sí, solamente con una supervisión mínima del docente para propiciar el protagonismo de los narradores como hacedores en primera persona de todo el proceso de escritura que tenía como objetivo en sí, el curso taller (redacción, corrección, edición, diseño e impresión). En este sentido se privilegió que esas correcciones fueran hechas de manera colaborativa y con el consentimiento de todos y todas, ello a través de medios virtuales de comunicación como el correo electrónico, un grupo de Facebook y la aplicación WhatsApp, ya que los tiempos para reunirse de forma presencial eran reducidos.

En ello se coincide con Pico y Rodríguez (2012, p. 9) quienes afirman:

“(...)Creemos que el valor del trabajo colaborativo responde a un modelo pedagógico que pone el acento en la interacción y la construcción colectiva de conocimientos, que sin duda se optimizan cuando se combinan con el trabajo en red. La colaboración en el contexto del aula invita a docentes y estudiantes a caminar juntos, sumando esfuerzos, talentos y competencias. Incentiva el aprender haciendo, el aprender interactuando, el aprender compartiendo. La riqueza de la colaboración también reside en que los estudiantes aprenden reflexionando sobre lo que hacen, ya que en el intercambio los 
saberes individuales se hacen explícitos y se tornan comprensibles para los demás. La capacidad para responder a demandas complejas y llevar a cabo adecuadamente diversas tareas supone una combinación de habilidades prácticas, conocimientos, motivaciones, valores, actitudes, emociones que se deben movilizar conjuntamente para lograr una acción eficaz. Contar con un caudal importante de competencias para trabajar con otros y colaborar en experiencias de aprendizaje es cada vez más necesario en las llamadas sociedades de la información y la comunicación (...)” (p. 9).

Siendo así, se consideran las narraciones publicadas en el libro de edición 2014

“(...)como pequeños aportes de situaciones pedagógicas reales a utilizar como insumos válidos y herramientas de comunicación que se pueden aplicar a la enseñanza para que otros docentes y directivos se vean reflejados en situaciones similares del contexto educativo en el que vivimos y crean que es posible que ellos también pueden narrar, sistematizar y documentar sus prácticas y actos de gestión (...)” (Tenaglia, 2014, p. 19).

\section{Conclusión}

Si hay algo que se debe reconocer en la didáctica actual con apoyo de las TIC, es que se ha podido salir de la linealidad del texto único. Hoy desde Internet se hace uso de la multimedialidad, la hipertextualidad, y la interactividad conjuntamente con la posibilidad de una comunicación sincrónica en tiempo real entre los actores educativos. Es importante tener en cuenta que sabiendo coordinar diferentes herramientas y recursos TIC, y con el aporte de los estudiantes por su condición de nativos digitales, se puede hacer de cada situación áulica una producción del conocimiento más participativa y democrática.

A su vez, el conocimiento histórico y más aún el reciente, es conocimiento que se debe construir no repetir, aspecto posible a través de las múltiples narraciones que se elaboran. En este proceso, se hace participar a diversos actores (docentes, directivos, estudiantes, etc). 
Con la nueva propuesta a desarrollar en el presente año, se pretende profundizar la experiencia previa, e incentivar y difundir el trabajo en el ámbito educativo en el uso de las narrativas como herramientas didácticas y de comunicación para la enseñanza.

La elección de producir narrativas sobre el uso de las nuevas tecnologías, como para la enseñanza de la historia contemporánea y reciente, demuestran que este género (el narrativo), usado como herramienta se puede aplicar en todos los ámbitos, niveles y/ o modalidades del sistema educativo y ser trabajado de manera transversal con cualquier aprendizaje o contenido, propiciando así, la democratización del conocimiento a partir de los relatos de los propios actores intervinientes en el sistema.

\section{REFERENCIAS}

Bombini, G. (2006). "Reinventar la Enseńanza de la Lengua y la Literatura”. Colección Formación Docente Serie Lengua y Literatura. Buenos Aires: Ediciones del Zorzal

Carnovale, V. (2007). "Aportes y problemas de los testimonios en la reconstrucción del pasado reciente en Argentina”, en Marina Franco y Florencia Levín (comps.): Historia Reciente. Perspectivas y desafíos para un campo en construcción. Buenos Aires: Paidós.

Castells, M. (2002). "La dimensión cultural de Internet” en Debates culturales. Tema: "Cultura y Sociedad del conocimiento: Presente y perspectivas del futuro" Universitat Oberta de Catalunya: Instituto de cultura.

Franco, M. y Levín, F. (2007). Historia reciente, perspectivas y desafíos para un campo en construcción. Buenos Aires: Paidós.

González, T. Mengo, R. Pizarro, H. Tenaglia, P. (2014). Desafíos para la enseñanza de las Ciencias Sociales en la era digital en Revista Educación y Humanidades. (3)2. p. 75. Temuco: Facultad de Educación y Humanidades. Universidad de la Frontera. 
Kriger, M. (2011). La enseñanza de la historia reciente como herramienta clave de la educación política. Persona y Sociedad (XXV)3. pp. 29-52. [en línea]. Recuperado de http:// www.personaysociedad.cl/la-ensenanza-de-la-historia-reciente-como-herramienta-clavede-la-educacion-politica/. ISNN 0716-730X

Pedró, F. (2012). La formación de aprendices para el nuevo milenio. Encuentro internacional de educación 2012-2013 ¿Cómo debería ser la educación en el siglo XXI? [en línea]. Recuperado de http://encuentro.educared.org/group/ cuales-son-los-principales-desafios-que-afronta-ho/page/francesc-pedro

Pico, L y Rodríguez, C. (2012). “Trabajo colaborativo” Serie estrategias en el aula para el modelo 1 a 1. Conectar Igualdad. p. 9. 1 ra Ed. Buenos Aires. Educ.ar S.E.

Prensky, M. (2001). Nativos Digitales, Inmigrantes Digital. From On the Horizon ( M C B University Press, (9)5, October 2001) [en línea]. Recuperado de http://psiytecnologia. files.wordpress.com/2010/02/prensky-nativos-digitales-inmigrantes-digital-traduccion. pdf

Suárez, D. (2005). La documentación narrativa de experiencias pedagógicas. Una estrategia para la formación de docentes. Documento realizado en el marco del proyecto "Estrategias y materiales pedagógicos para la Retención Escolar”. Buenos Aires: Ministerio de Educación Ciencia y Tecnología de la Nación y financiado por la OEA.

Sunkel, O. (2008). La precaria sostenibilidad de la democracia en Latinoamérica. Cuadernos del Cendes, 25(68), pp. 1-27

Tenaglia, P. (2014). Narrativas de experiencias pedagogicas significativas como herramientas de comunicación para la enseńanza en el contexto de la escuela TIC. Aportes desde la práctica docente y gestión directiva. Córdoba: Editorial Investigación. 